\title{
Three-Dimensional Heat Transfer Analysis of a TG-CVI Reactor
}

\author{
Zaher Ramadan ${ }^{1}$, Yeonho Choi ${ }^{2}$, Jeongmin Lee ${ }^{2}$, Dongwon Im ${ }^{2}$, Ik-Tae Im ${ }^{1, a}$ \\ 1 Chonbuk National University, Jeonju, Republic of Korea \\ ${ }^{2}$ Dacc Carbon, Jeonju, Republic of Korea
}

\begin{abstract}
Thermal-gradient chemical vapor infiltration (TG-CVI) is an alternative process to the classical CVI by involving a temperature gradient to obtain uniform densification. It allows fabricating $\mathrm{C} / \mathrm{SiC}$ and $\mathrm{C} / \mathrm{C}$ composites starting from a fibrous preform and gasses precursor. The main interest of these processes is increasing the density homogeneity and the densification rate. In the proposed TG-CVI reactor, a heater with a constant temperature (1323 $\mathrm{K}$ ) is placed in the core of the reactor and the cool gas flows from the outside to the center. Therefore, a continuous radially moving densification from inside to the outside of porous preform can be achieved. A numerical model was developed in order to investigate temperature distribution and velocity profile in the TG-CVI reactor. The commercial CFD software CFD-ACE+ was used. The obtained three-dimensional results were compared with experimental data. Three reference points were defined to measure the temperature in the preform. The computed results of TG-CVI showed a good agreement with the measured data. The simulation results of this study are important for the optimization of the densification process in the preform in The TG-CVI reactor.
\end{abstract}

\section{Introduction}

Chemical vapour infiltration (CVI) is considered as one of the main processes used to densify porous preform for carbon/carbon and carbon/silicon carbide composites $[1,2]$. In this process, precursor gasses are transported into the porous preform. While the infiltration is taking place, the deposition occurs in the preform gradually. There are several kinds of CVI such as isothermal, forced flow CVI (F-CVI) and thermal gradient CVI process. The first implementation of CVI was carried out under isothermal conditions (I-CVI)[3]. This type is the simplest type of CVI in which the preform is placed in a uniform temperature furnace. The deposition in I-CVI starts from the exterior surface of the preform. Usually, the process is conducted at low temperature and pressure to avoid the sealing of porous preform [4]. As a result, the densification rate is low, the consumed time to achieve the process is extremely high, and this process is energy-consuming.

These drawbacks can be avoided by thermal-gradient CVI (TG-CVI) process [5]. The heater is located in the center of the reactor and the deposition starts from the interior surface. During the proceeding of the infiltration, the deposition moves gradually to the outside surface overcoming the sealing of porous which happens in ICVI process. The infiltration time becomes shorter and the deposition becomes more uniform [6].

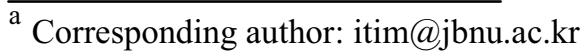

In this paper, a computational fluid dynamic (CFD) study has been carried out to simulate temperature distribution and velocity pattern in a 3 dimensional TGCVI reactor.

\section{Numerical Analysis}

The schematic of a TG-CVI reactor considered for simulation in the study is shown in Figure 1. This CVI reactor is used in the industrial fabrication of silicon carbide by introducing a mixture of methyltichlorosilane (MTS) and hydrogen to the reactor. In this stage of study, hydrogen gas in the experimental and the numerical study was used to investigate the temperature and fluid profile regardless to the chemical reaction and deposition. The heat source is a cylindrical heater located in the center of the reactor. It is electrically heated to maintain a constant temperature equals to $1323 \mathrm{~K}$. Hydrogen gas is supplied from the bottom of the reactor and starts to infiltrate porous medium of the preform. The cylindrical preform is composed of carbon reinforced fibres.

The first step of the simulation is to build a threedimensional model based on the reactor which has been setup for process design experiments. The preprocessor which called CFD-Geom , which is provided by the commercial CFD software was used to construct the three dimensional grid of the reactor for numerical simulation. Two different grid systems were created to validate the independent of the size of the mesh cells. Figure 2 shows the created three dimensional model of the CVI reactor which is meshed with tetrahedral 
elements. The first one shown in Figure 2(a) is divided by a total of 500,400 cells and the second dense grid shown in Figure 2(b) is divided by a total of 750,521 cells.

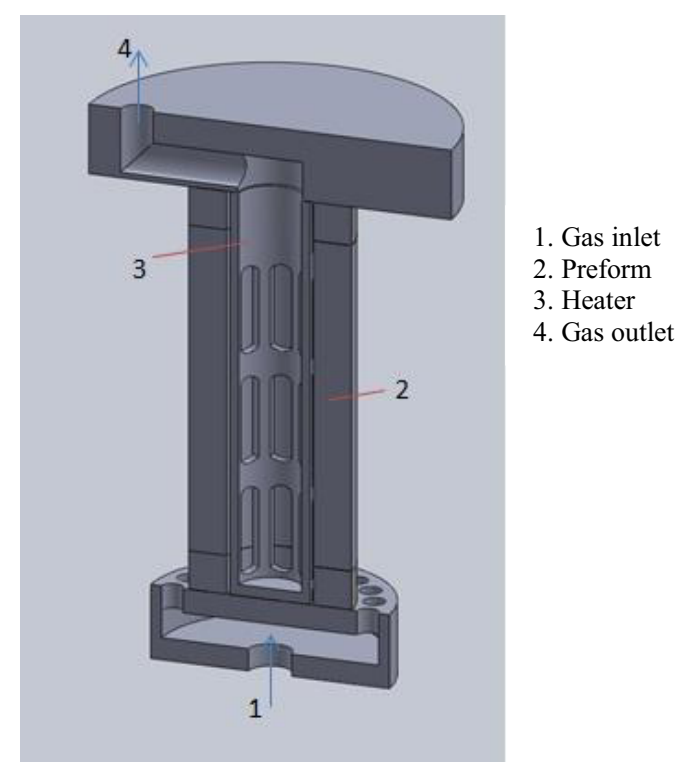

Figure 1. Schematic of the CVI reactor

CFD-ACE + was used to compute velocity and temperature profiles in the reactor. A fixed mass flow rate and a constant temperature of $300 \mathrm{~K}$ were used in the inlet boundary condition. At the outlet, the gas pressure was set at a fixed value of 20 torr. A no-slipping boundary condition was adopted at the reactor walls and preform surface. The heater temperature was set to 1323 K. A discrete ordinate method (DOM) was used to calculate radiation heat transfer. Porosity throughout the preform is set to fixed value of 0.7 in this study. Properties of gas were allowed to vary in piecewise linear with the temperature. Convergence criterion of $1 \times 10-4$ was applied to the residual of continuity, momentum, and energy equations. All simulations were performed under steady state conditions and calculation was continued until the convergence was achieved.

Table 1. TG-CVI operating conditions

\begin{tabular}{lcl}
\hline Operating Conditions & & \\
\hline Heater temperature & 1323 & {$[\mathrm{~K}]$} \\
Ambient temperature & 300 & {$[\mathrm{~K}]$} \\
$\mathrm{H}_{2}$ flow rate & 3960 & {$[\mathrm{ml} / \mathrm{min}]$} \\
Pressure & 20 & {$[$ torr $]$} \\
Porosity & 0.7 & \\
\hline
\end{tabular}

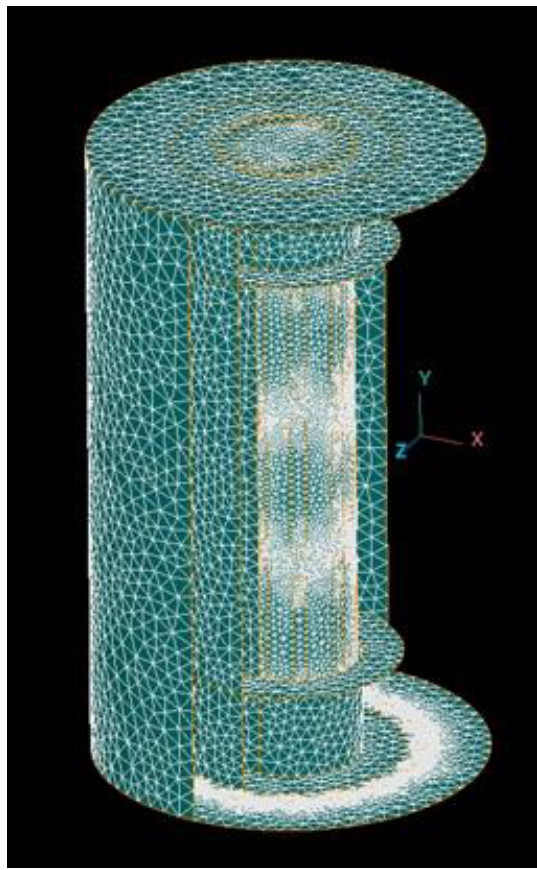

(a) Moderate grid, 535,713 cells

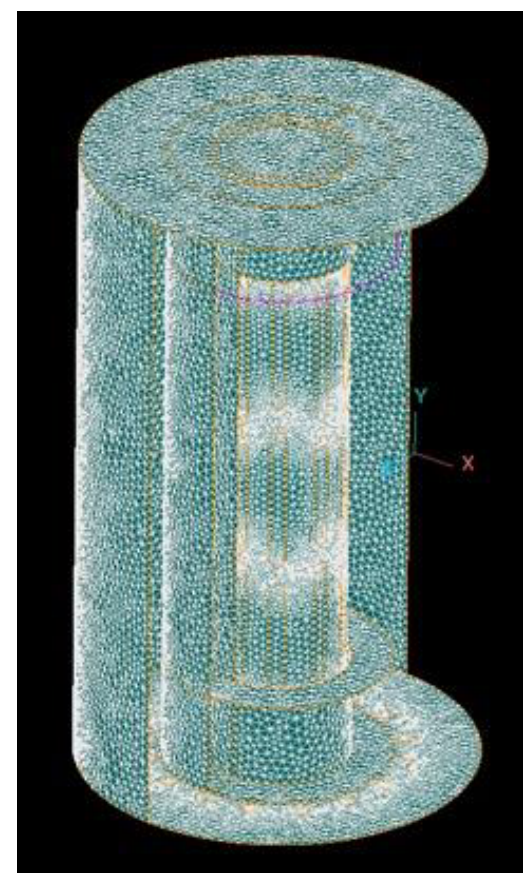

(b) Dense grid, 779,173 cells

Figure 2. Two different sizes of grid systems for numerical simulation

\section{Results and discussion}

The validation of numerical calculation was performed by comparing temperature at several points between the measured data and the numerical solution results. 
The computed and measured data are in a good agreement as shown in Figure 3. Three thermocouples were used in the experiment and placed in specific positions to show the temperature gradient along the preform length and thickness as shown in Figure 3. Table 2 shows the error between the simulation and experimental results.

Table 2. Computed and measured temperature

\begin{tabular}{cccc}
\hline Point & $\begin{array}{c}\text { Measured } \\
\text { temperature } \\
{[\mathrm{K}]}\end{array}$ & $\begin{array}{c}\text { Computed } \\
\text { temperature } \\
{[\mathrm{K}]}\end{array}$ & $\begin{array}{c}\text { Relative } \\
\text { error \% }\end{array}$ \\
\hline $\mathrm{T}_{1}$ & 1323 & 1294 & 2 \\
$\mathrm{~T}_{2}$ & 929 & 970 & 4 \\
$\mathrm{~T}_{3}$ & 794 & 763 & 3.7 \\
\hline
\end{tabular}

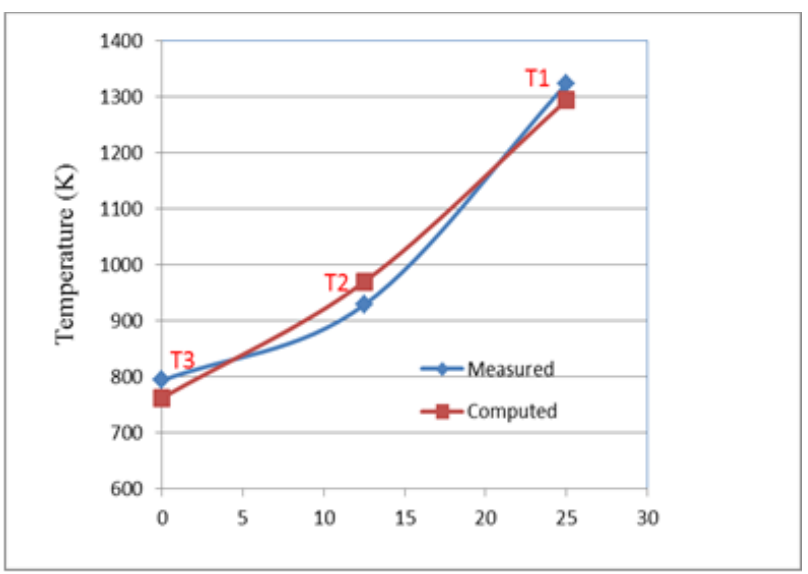

(a) Comparison between computed and measured data

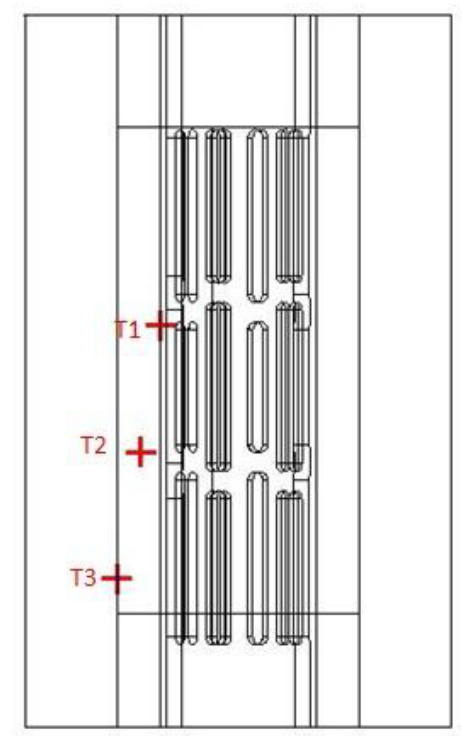

(b) Points for comparison

Figure 3. Computed and measured temperature at three points in the preform
Figure 4 illustrates temperatures distribution in the reactor. A gradually decreasing in temperatures starting from the heater wall toward the reactor wall is noticed. However there is a significant drop in temperatures in the bottom of the reactor near the inlet region. It is seen from Figure 4 that the temperature distribution across the preform block is almost uniform. It is also seen that the temperature drop across the preform thickness is much higher than the temperature drop along the preform height.

The numerical simulation provides more details about the temperature distribution. Figure 5 illustrates the temperature profile around the outside surface of the preform. The temperature fluctuates between $744 \mathrm{~K}$ and $763 \mathrm{~K}$. Figure 6 shows the value of T2 in two different positions with same radius. The values are $986 \mathrm{~K}$ and 970 $\mathrm{K}$ for $\mathrm{T} 2 \mathrm{a}$ and $\mathrm{T} 2 \mathrm{~b}$, respectively. The slight temperature difference in the same radius of the preform is due to the heater design and gas flow. The fluctuation of the temperature occurs due to the flow of the gasses in the heater slots.

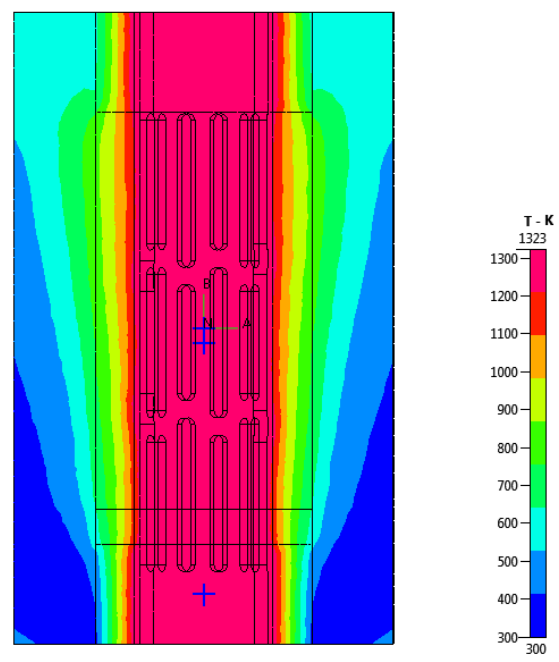

Figure 4. Temperature distribution in a cross section of the TG-CVI reactor

Figure 7 represents the flow pattern in the reactor. It is important to note the increasing of velocity inside the heater. The flow faces a large resistance due to the porous medium. After penetrating the preform in addition to the resistance decreasing, the relatively high temperature causes the increasing in velocity. The velocity vectors shown in Figure7 (a) have been colored and sized in proportion to their magnitude. However, in Figure 7(b) the velocity vectors size has been modified to clarify the flow streamlines. 


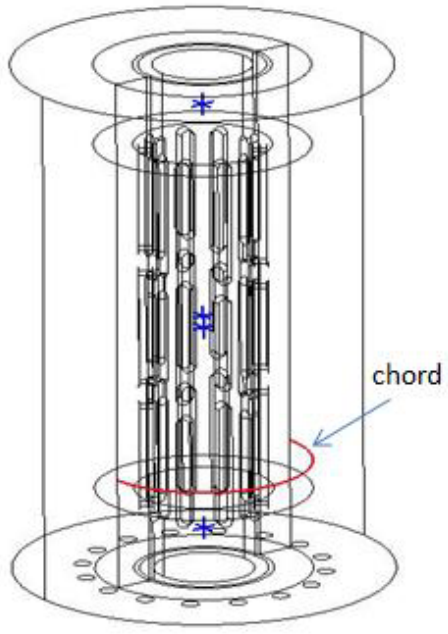

(a) Chord in $T_{3}$ position

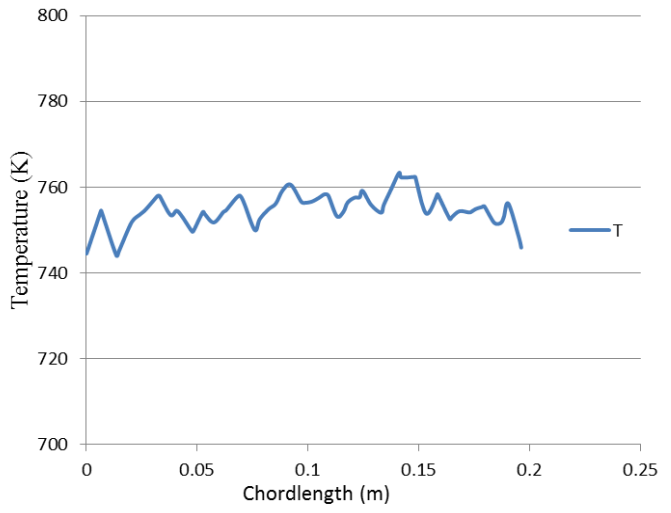

(b) Temperature variation

Figure 5. Temperature distribution on the surface of the preform points in the preform
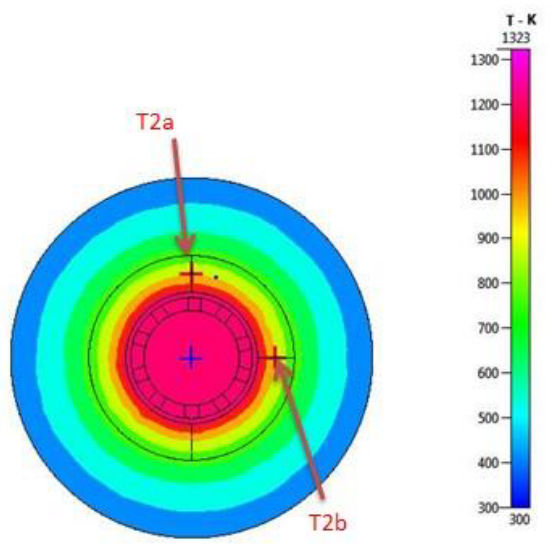

Figure 6. Temperature distribution at the cross section at $\mathrm{T}_{2}$ position (a)

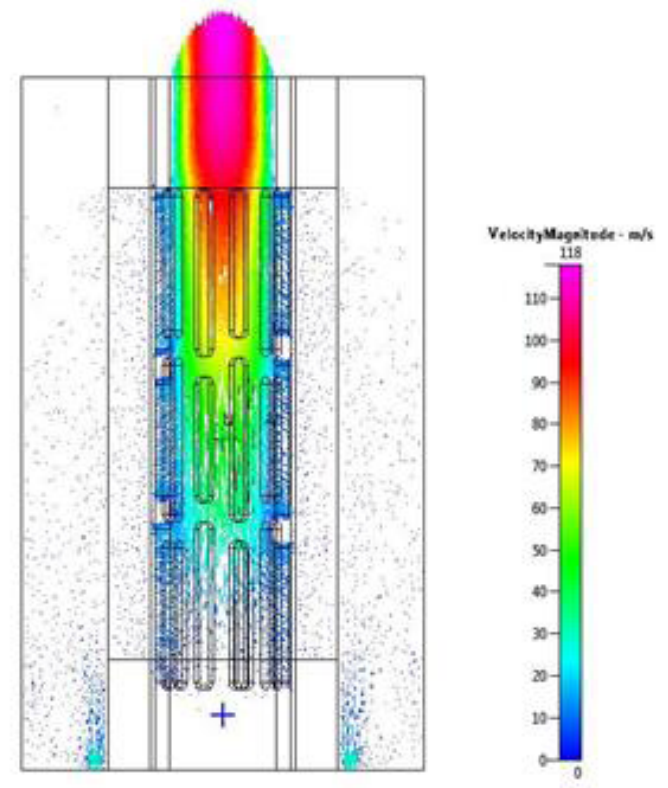

(b)

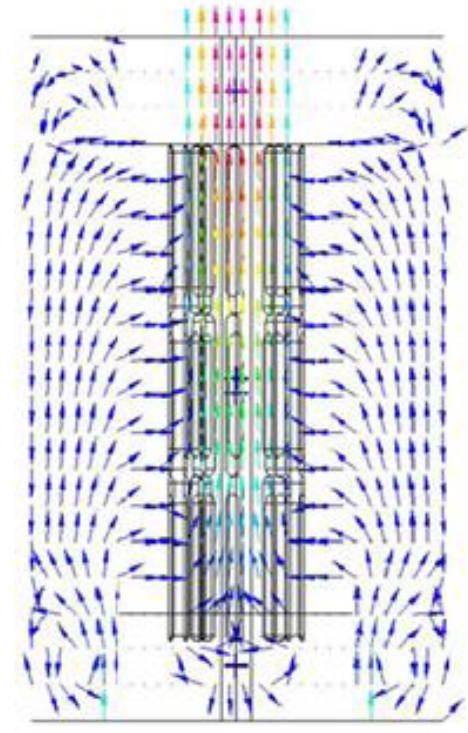

Figure 7. Flow pattern in the reactor, (a) real magnitude, (b) using vectors with the same magnitude

\section{Summary}

A three dimensional model was developed to simulate temperature and velocity profile in a TG-CVI reactor. An approximately uniform temperature profile was observed in the preform. The temperature drop along the preform was low, whereas the decreasing in temperature field across the preform thickness was relatively high. The simulation results of this study are important for the optimization of the TG-CVI operating conditions to achieve uniform and high deposition rate. 


\section{Acknowledgements}

This work (Grant No. C0250033) was supported by Business for Cooperative R\&D between Industry, Academy, and Research Institute funded Korea Small and Medium Business Administration in 2014

\section{References}

1. G. Y. Chung, B. J. McCoy, J. M. Smith, D. E. Cagliostro, and M. Carswell, 'Chemical Vapor Infiltration: Modelling Solid Matrix Deposition in Ceramic-Ceramic Composites', Chemical Engineering Science, 46 (1991), pp. 723-33.

2. R. Naslain and F. Langlais, "Fundamental and Practical Aspects of the Chemical Vapor Infiltration of Porous Substrates," Carbon, 27 (1990), pp. 221-35.
3. R. Fedous, R. Naslain and F. Langlais, A model for the isothermal isobaric CVI in straight cylindrical pore. Application to the CVI of SiC. Journal of Materials Synthesis and Processing 1(2), (1993), pp.61-74.

4. J. G. Zhao, K. Z. Li, H. J. Li and C. Wang, The influence of thermal gradient on pyrocarbon deposition in carbon/carbon composites during the CVI process, Carbon, 44 (2006), pp. 786-791.

5. K. Jiang, H. Li, and M. Wang, The numerical simulation of the thermal-gradient CVI process on positive pressure condition, Material Letters, 54 (2004), pp. 419-423.

6. Tago, T., Kawase, M., Ikuta, Y., Hashimoto, K. Numerical simulation of the thermal-gradient chemical vapor infiltration process for production of fibre-reinforced ceramic composite, Chemical Engineering Science 56 (2001), pp. 2161-2170. 